\title{
Second-Order Correct Boundary Conditions for the Numerical Solution of the Mixed Boundary Problem for Parabolic Equations
}

\section{By George W. Batten, Jr.}

1. Introduction. Consider the parabolic equation

(1) $\frac{\partial^{2} u}{\partial x^{2}}-a(x, t) \frac{\partial u}{\partial t}+b(x, t) \frac{\partial u}{\partial x}+c(x, t) u=d(x, t), 0<x<1,0<t \leqq T$, and the initial condition

$$
u(x, 0)=f(x), \quad 0 \leqq x \leqq 1 .
$$

Assume that $a(x, t)>0$. It is well known (Douglas [1], Rose [ $[$ ]) that the Dirichlet problem (1), (2), with boundary conditions

$$
\left\{\begin{array}{l}
u(0, t)=g(t), \\
u(1, t)=h(t),
\end{array} \quad 0<t \leqq T,\right.
$$

can be approximated by the solution of the difference equation

$$
\begin{aligned}
& \Delta_{x}^{2} w_{i n}-a_{i n} \Delta_{t} w_{i n}+b_{i n} \Delta_{x} w_{i n} \\
& \quad+c_{i n} w_{i n}=d_{i n}, \quad i=1, \cdots, I-1, n=1, \cdots, N,
\end{aligned}
$$

subject to the initial condition

$$
w_{i 0}=f_{i}, \quad i=0, \cdots, I,
$$

and the boundary conditions

$$
\left\{\begin{array}{l}
w_{0 n}=g_{n}, \\
w_{\text {In }}=h_{n},
\end{array} \quad n=1, \cdots, N .\right.
$$

The subscripts $i$ and $n$ indicate that the function is evaluated at the point $(i h, n k)$ where $h=I^{-1}, k=T N^{-1}$. The difference operators in (4) are defined by

$$
\left\{\begin{array}{l}
\Delta_{x}^{2} w_{i n}=\frac{1}{h^{2}}\left(w_{i-1, n}-2 w_{i n}+w_{i+1, n}\right) \\
\Delta_{t} w_{i n}=\frac{1}{k}\left(w_{i n}-w_{i, n-1}\right) \\
\Delta_{x} w_{i n}=\frac{1}{2 h}\left(w_{i+1, n}-w_{i-1, n}\right) .
\end{array}\right.
$$

If $^{*} u \varepsilon C^{4,2}([0,1] \times[0, T])$, then the error

Received June 21, 1962. This research was supported by the Air Force Office of Scientific Research. In May, 1961 the author submitted a thesis containing the principle part of this paper to the Rice University in partial fulfillment of the requirements for a degree of Master of Arts.

${ }^{*} \varphi(x, t) \varepsilon C^{\alpha, \beta}(R)$ if and only if $\varphi$ is continuously differentiable $\alpha$ times with respect to $x$ and $\beta$ times with respect to $t$ in the region $R$. 


$$
z_{i n}=u_{i n}-w_{i n}
$$

satisfies

$$
\max _{i, n}\left|z_{i n}\right|=O\left(h^{2}+k\right) .
$$

If the conditions (3) are replaced by the Neumann conditions

$$
\left\{\begin{array}{l}
\frac{\partial u}{\partial x}(0, t)=g(t) \\
\frac{\partial u}{\partial x}(1, t)=h(t)
\end{array}\right.
$$

$$
0<t \leqq T
$$

then the solution $w_{i n}$ of equations (4) and (5) with boundary conditions

$$
\begin{cases}\frac{w_{1 n}-w_{0 n}}{h}=g_{n}, & n=1, \cdots, N, \\ \frac{w_{I n}-w_{I-1, n}}{h}=h_{n}, & n=1, \cdots, N,\end{cases}
$$

converges to $u_{i n}$, but the error is $O(h+k)$ (Douglas [3]). From the analysis, it is clear that the $h$ (instead of $h^{2}$ ) arises in the first order correctness of the boundary conditions.

Recently, Isaacson [4] has shown that an approximation that is second order correct in $h$ can be obtained by replacing conditions (11) with

$$
\begin{cases}\frac{w_{1 n}-w_{-1, n}}{2 h}=g_{n}, & n=1, \cdots, N, \\ \frac{w_{I+1, n}-w_{I-1, n}}{2 h}=h_{n}, & n=1, \cdots, N .\end{cases}
$$

This result is not entirely pleasing, however, for it requires the assumption that $u$ can be extended to satisfy sufficient continuity conditions in $[-h, 1+h] \times[0, T]$.

2. Interior Approximations. In the present paper, it is shown that if the centered differences in (12) are replaced by one-sided, second order correct differences, the error is $O\left(h^{2}+k\right)$. This result applies (as do those mentioned above) if the Neumann conditions (10) are replaced by the mixed boundary conditions

$$
\begin{array}{ll}
-p(t) u(0, t)+q(t) \frac{\partial u}{\partial x}(0, t)=g(t), & 0<t \leqq T, \\
-r(t) u(1, t)-s(t) \frac{\partial u}{\partial x}(1, t)=h(t), & 0<t \leqq T .
\end{array}
$$

It is necessary to assume that $p, q, r$, and $s$ are non-negative, and that $p+q$ and $r+s$ are bounded away from zero. It is not necessary to assume, as do both Isaacson [4] and Rose [6], that one or more of the coefficients $p, q, r, s$ is bounded away from zero.

Assume that the quantities $a, b, c, d, p, q, r$, and $s$ are bounded, and that 
$u \varepsilon C^{4,2}([0,1] \times[0, T])$. By Taylor's theorem

$$
\begin{aligned}
& \Delta_{x}^{2} u_{i n}-a_{i n} \Delta_{t} u_{i n}+b_{i n} \Delta_{x} u_{i n} \\
& +c_{i n} u_{i n}=d_{i n}+A_{i n}, \quad i=1, \cdots, I-1, n=1, \cdots, N,
\end{aligned}
$$

where $\left|A_{i n}\right|<A\left(h^{2}+k\right)$ and $A$ is a constant. Similarly,

$$
\left\{\begin{array}{l}
\frac{1}{2 h}\left(-3 u_{0 n}+4 u_{I n}-u_{2 n}\right)=\left.\frac{\partial u}{\partial x}\right|_{0 n}+{B_{n}}^{+}, \\
\frac{1}{2 h}\left(u_{I-2, n}-4 u_{I-1, n}+3 u_{I n}\right)=\left.\frac{\partial u}{\partial x}\right|_{I n}+{B_{n}}^{-},
\end{array}\right.
$$

where $B_{n}{ }^{+}$and $B_{n}{ }^{-}$are bounded by a constant multiple of $h^{2}$. For simplicity let

$$
\left\{\begin{array}{l}
\Delta_{x}^{+} u_{0 n}=\frac{1}{2 h}\left(-3 u_{0 n}+4 u_{1 n}-u_{2 n}\right) \\
\Delta_{x}^{-} u_{I n}=\frac{1}{2 h}\left(u_{I-2, n}-4 u_{I-1, n}+3 u_{I n}\right) .
\end{array}\right.
$$

Then

$$
\left\{\begin{array}{l}
-p_{n} u_{0 n}+q_{n} \Delta_{x}{ }^{+} u_{0 n}=g_{n}+B_{0 n}, \\
-r_{n} u_{I n}-s_{n} \Delta_{x}{ }^{-} u_{I n}=h_{n}+B_{I n},
\end{array} \quad n=1, \cdots, N,\right.
$$

where $\left|B_{\text {in }}\right| \leqq B h^{2}$ and $B$ is a constant.

Approximate $u_{i n}$ by the solution $w_{i n}$ of (4) and (5) with boundary conditions

$$
\left\{\begin{array}{l}
-p_{n} w_{0 n}+q_{n} \Delta_{x}{ }^{+} w_{0 n}=g_{n}, \\
-r_{n} w_{i n}-s_{n} \Delta_{x}{ }^{-} w_{i n}=h_{n},
\end{array} \quad n=1, \cdots, N .\right.
$$

Then the error (8) satisfies

$$
\left\{\begin{aligned}
\Delta_{x}^{2} z_{\text {in }}-a_{i n} \Delta_{t} z_{i n}+b_{i n} \Delta_{x} z_{i n}+c_{i n} z_{i n} & =A_{\text {in }}, & i & =1, \cdots, I-1, \\
-p_{n} z_{0 n}+q_{n} \Delta_{x}{ }^{+} z_{0 n} & =B_{0 n}, & n & =1, \cdots, N, \\
-r_{n} z_{I n}-s_{n} \Delta_{x}{ }^{-} z_{I n} & =B_{I n}, & n & =1, \cdots, N, \\
z_{i 0} & =0, & i & =1, \cdots, I-1 .
\end{aligned}\right.
$$

In order to bound $z_{\text {in }}$ we prove the following lemmas.

Lemma 1. Let $v_{\text {in }}$ satisfy

$$
\left\{\begin{aligned}
\Delta_{x}^{2} v_{i n}-a_{i n} \Delta_{t} v_{i n}+b_{i n} \Delta_{x} v_{i n}+c_{i n} v_{i n} \leqq 0, & & i=1, \cdots, I-1, \\
-p_{n} v_{0 n}+q_{n} \Delta_{x}{ }^{+} v_{0 n} \leqq 0, & n & =1, \cdots, N, \\
-r_{n} v_{I n}-s_{n} \Delta_{x}{ }^{-} v_{I n} \leqq 0, & n & =1, \cdots, N, \\
v_{i 0} \geqq 0, & n & =1, \cdots, N, \\
& i & =1, \cdots, I-1 .
\end{aligned}\right.
$$


If, for all $i=1, \cdots, I-1, n=1, \cdots, N$,

$$
\left\{\begin{aligned}
0<\alpha & \leqq a_{i n}, \\
\left|b_{i n}\right|<\beta & \leqq \frac{1}{h}, \\
0 \leqq-c_{i n}<\gamma & \leqq \frac{\alpha}{k} \\
p_{n}, q_{n}, r_{n}, s_{n} & \geqq 0, \\
p_{n}+q_{n} & >0, \\
r_{n}+s_{n} & >0,
\end{aligned}\right.
$$

where $\alpha, \beta, \gamma$ are constants, then

$$
v_{\text {in }} \geqq 0, \quad i=1, \cdots, I-1, n=1, \cdots, N .
$$

Note that $v_{\text {in }}$ is non-negative only in the interior of the region. With little difficulty, one can construct examples for which (22) holds, but for which $v_{0 n}<0$ and $v_{1 n}<0$, for some $n$.

Proof. Suppose the lemma is false. Let

$$
n_{0}=\min \left\{n \mid v_{\text {in }}<0 \text { for some } i, \quad 1 \leqq i \leqq I-1\right\} .
$$

Then $n_{0} \geqq 1$. Let $i_{0}$ denote a value such that $v_{i_{0}, n_{0}}$ is a local negative minimum with respect to $i$. There are three cases.

Case 1: $i_{0}=0$. Since $v_{0, n_{0}}<0$,

$$
0 \geqq-p_{n_{0}} v_{0, n_{0}}+q_{n_{0}} \Delta_{x}{ }^{+} v_{0, n_{0}} \geqq q_{n_{0}} \Delta_{x}{ }^{+} v_{0, n_{0}} .
$$

If $q_{n_{0}}=0$, then $p_{n_{0}}>0$ and it follows that $v_{0, n_{0}} \geqq 0$, contradicting the hypothesis. Thus $q_{n_{0}}>0$, and by $(24)$,

$$
\Delta_{x}^{+} v_{0, n_{0}} \leqq 0
$$

Therefore,

$$
\begin{aligned}
\Delta_{x}^{2} v_{1, n_{0}} & =\frac{1}{h^{2}}\left(v_{0, n_{0}}-2 v_{1, n_{0}}+v_{2, n_{0}}\right) \\
& =\frac{1}{h}\left[\frac{1}{2 h}\left(v_{2, n_{0}}-v_{0, n_{0}}\right)-\Delta_{x}^{+} v_{0, n_{0}}\right] \\
& \geqq \frac{1}{h}\left[\frac{1}{2 h}\left(v_{2, n_{0}}-v_{0, n_{0}}\right)\right] \\
& =\frac{1}{h} \Delta_{x} v_{1, n_{0}} .
\end{aligned}
$$

From the second of conditions (21),

$$
\Delta_{x}^{2} v_{1, n_{0}} \geqq-b_{i n} \Delta_{x} v_{1, n_{0}} .
$$

Thus, the first of inequalities (20) gives

$$
-a_{1, n_{0}} \Delta_{t} v_{1, n_{0}}+c_{1, n_{0}} v_{1, n_{0}} \leqq 0 .
$$

Since $c_{1, n_{0}} \leqq 0$ and $v_{1, n_{0}-1} \geqq 0$, it follows that $v_{1, n_{0}} \geqq 0$. 
Case 2: $i_{0}=I$. By an argument analogous to that of case 1, it follows that $v_{l-1, n_{0}} \geqq 0$.

Case $3: 1 \leqq i_{0} \leqq I-1$. Here a maximum principle argument is used. From the first of inequalities $(20)$,

$$
\begin{aligned}
\left(\frac{1}{k} a_{i_{0}, n_{0}}-c_{i_{0}, n 0}\right) & v_{i_{0}, n_{0}} \geqq\left(\frac{1}{h^{2}}-\frac{1}{2 h} b_{i_{0}, n_{0}}\right)\left(v_{i_{0}-1, n_{0}}-v_{i_{0}, n_{0}}\right) \\
+ & \left(\frac{1}{h^{2}}+\frac{1}{2 h} b_{i_{0}, n_{0}}\right)\left(v_{i_{0}+1, n_{0}}-v_{i_{0}, n_{0}}\right)+\frac{1}{k} a_{i_{0}, n_{0}} v_{i_{0}, n_{0}-1} .
\end{aligned}
$$

Since every term on the right is non-negative, it follows that $v_{i_{0}, n_{0}} \geqq 0$. This is a contradiction.

LEMMA 2. Under conditions (21) and the conditions

Q. E. D.

i) for some $\delta$,

$$
\frac{1}{4} p_{n}+q_{n} \geqq \delta>0 \text { and } \frac{1}{4} r_{n}+s_{n} \geqq \delta>0,
$$

ii) $k<\frac{\alpha}{4 \gamma}$,

there exists a funtcion $\zeta(x, t)$ such that

$$
\left\{\begin{array}{rlrl}
\Delta_{x}{ }^{2} \zeta_{i n}-a_{i n} \Delta_{t} \zeta_{\text {in }}+b_{i n} \Delta_{x} \zeta_{\text {in }}+c_{i n} \zeta_{\text {in }} \leqq-1, & i & =1, \cdots, I-1, \\
& n=1, \cdots, N, \\
-p_{n} \zeta_{0 n}+q_{n} \Delta_{x}{ }^{+} \zeta_{0 n} \leqq-1, & n & =1, \cdots, N, \\
-r_{n} \zeta_{\text {In }}-s_{n} \Delta_{x}{ }^{-} \zeta_{\text {In }} \leqq-1, & n & =1, \cdots, N, \\
\zeta_{i 0} \geqq 0, & i & =1, \cdots, I-1,
\end{array}\right.
$$

and

$$
0 \leqq \zeta(x, t) \leqq M_{0}, \quad 0 \leqq x \leqq 1,0 \leqq t \leqq T,
$$

where $M_{0}$ is a constant depending on $\alpha, \beta, \gamma, \delta$ and $T$.

Proof. Let

$$
\zeta^{*}(x, t)=\left(\frac{1}{2}-x\right)^{2} .
$$

Then

$$
\begin{array}{r}
\Delta_{x}^{2} \zeta_{i n}^{*}-a_{i n} \Delta_{t} \zeta_{i n}^{*}+b_{i n} \Delta_{x} \zeta_{i n}^{*}+c_{i n} \zeta_{i n}^{*}=2-2 b_{i n}\left(\frac{1}{2}-x_{i}\right) \\
+c_{i n}\left(\frac{1}{2}-x_{i}\right)^{2} \leqq 2+\beta+\frac{1}{4} \gamma, \quad i=1, \cdots, I-1 \\
n=1, \cdots, N
\end{array}
$$

and

$$
\left\{\begin{array}{l}
-p_{n} \zeta_{0 n}^{*}+q_{n} \Delta_{x}{ }^{+} \zeta_{0 n}^{*}=-\frac{1}{4} p_{n}-q_{n} \leqq-\delta, \\
-r_{n} \zeta_{I n}^{*}-s_{n} \Delta_{x}{ }^{-} \zeta_{I n}^{*}=-\frac{1}{4} r_{n}-s_{n} \leqq-\delta,
\end{array} \quad n=1, \cdots, N .\right.
$$

Let $\zeta^{* *}(x, t)=e^{\sigma t}, \sigma>0$. Then

$$
\begin{aligned}
& \Delta_{x}^{2} \zeta^{* *}{ }_{i n}-a_{i n} \Delta_{t} \zeta^{* *}{ }_{i n}+b_{i n} \Delta_{x} \zeta^{* *}{ }_{i n}+c_{i n} \zeta^{* *}{ }_{i n} \\
& \quad=e^{\sigma t_{n}}\left[-\frac{1}{k} a_{i n}\left(1-e^{-\sigma k}\right)+c_{i n}\right] \leqq e^{\sigma t_{n}}\left[-\frac{\alpha}{k}\left(1-e^{-\sigma k}\right)+\gamma\right] .
\end{aligned}
$$


By Taylor's theorem,

$$
e^{-\sigma k}=1-\sigma k+\frac{\sigma^{2} k^{2}}{2} e^{-\sigma k^{\prime}}<1-\sigma k+\frac{\sigma^{2} k^{2}}{2}
$$

where $0<k^{\prime}<k$. Since $k<\frac{1}{4} \alpha \gamma^{-1}$, for $\sigma=2 \alpha^{-1} \gamma$ it follows that

$$
\Delta_{x}^{2} \zeta_{i n}^{* *}-a_{i n} \Delta_{t} \zeta_{i n}^{* *}+b_{i n} \Delta_{x} \zeta_{i n}^{* *}+c_{i n} \zeta_{i n}^{* *}<-e^{\sigma t_{n}} \frac{\gamma}{2}<-\frac{\gamma}{2}<0
$$

Also,

$$
\left\{\begin{array}{l}
-p_{n} \zeta_{0 n}^{* *}+q_{n} \Delta_{x}^{+} \zeta_{0 n}^{* *}=-p_{n} e^{\sigma t_{n}} \leqq 0 \\
-r_{n} \zeta_{0 n}^{* *}-s_{n} \Delta_{x}{ }^{-} \zeta_{0 n}^{* *}=-r_{n} e^{\sigma t_{n}} \leqq 0
\end{array}\right.
$$

Let $M_{1}$ and $M_{2}$ be constants satisfying

$$
\left\{\begin{array}{l}
M_{1} \geqq \frac{1}{\delta} \\
M_{2} \geqq \frac{2}{\gamma}\left[1+M_{1}\left(2+\beta+\frac{1}{4} \gamma\right)\right] .
\end{array}\right.
$$

Then

$$
\zeta=M_{1} \zeta^{*}+M_{2} \zeta^{* *}
$$

satisfies the conditions of the lemma.

Q. E. D.

TheOREM 1. If $u \varepsilon C^{4,2}([0,1] \times[0, \mathrm{~T}])$ is a solution of $(1)$ with initial condition (2) and boundary conditions (13), if there exist constants $\alpha, \beta, \gamma, \delta$ such that

$$
\left\{\begin{array}{lr}
0<\alpha \leqq a(x, t), & 0<x<1,0<t \leqq T, \\
|b(x, t)|<\beta, & 0<x<1,0<t \leqq T, \\
0 \leqq-c(x, t)<\gamma, & 0<x<1,0<t \leqq T, \\
p(t), q(t), r(t), s(t) \geqq 0, & 0<t \leqq T, \\
p(t)+q(t) \geqq \delta>0, & 0<t \leqq T, \\
r(t)+s(t) \geqq \delta>0, & 0<t \leqq T,
\end{array}\right.
$$

and if $h$ and $k$ are sufficiently small, then

$$
\max _{0 \leqq i \leqq I}\left|z_{\text {in }}\right| \leqq M\left(h^{2}+k\right), \quad n=1, \cdots, N
$$

where $M$ is a constant that depends on $\alpha, \beta, \gamma, \delta, T$.

Proof. Let $\zeta(x, t)$ be a function given by Lemma 2 .

Let $M_{3}=\max (A, B)$ and let

$$
\left\{\begin{array}{lrl}
v_{i n}^{+}=M_{3}\left(h^{2}+k\right) \zeta_{i n}+z_{i n}, & i=0, \cdots, I \\
v_{i n}^{-}=M_{3}\left(h^{2}+k\right) \zeta_{i n}-z_{i n}, & n=0, \cdots, N .
\end{array}\right.
$$

By (19) if $h$ and $k$ are so small that $\beta \leqq h^{-1}, \gamma \leqq \alpha k^{-1}$, and $k<\frac{1}{4} \alpha \gamma^{-1}$, then $v_{i n}^{+}$and 
$\overrightarrow{v_{\text {in }}}$ satisfy the conditions of Lemma 1 . Hence

$$
\begin{cases}v_{i n}^{+} \geqq 0, & i=1, \cdots, I-1, \\ v_{i n}^{-} \geqq 0, & n=1, \cdots, N,\end{cases}
$$

whence

$$
\begin{aligned}
\left|z_{\text {in }}\right| \leqq M_{0} M_{3}\left(h^{2}+k\right), & i=1, \cdots, I-1 \\
n & =1, \cdots, N .
\end{aligned}
$$

From (19),

$$
\begin{aligned}
& z_{0 n}=\left(-p_{n}-\frac{3}{2 h} q_{n}\right)^{-1}\left[\frac{q_{n}}{2 h}\left(z_{2 n}-4 z_{1 n}\right)+B_{0 n}\right] \\
& =-\left(2 h p_{n}+3 q_{n}\right)^{-1}\left[q_{n}\left(z_{2 n}-4 z_{1 n}\right)+2 h B_{0 n}\right] \\
& \leqq M_{4}\left[\max \left(\left|z_{1, n}\right|,\left|z_{2, n}\right|\right)+h^{3}\right], \quad n=1, \cdots, N \text {, }
\end{aligned}
$$

where $M_{4}$ is a constant. A similar inequality holds for $z_{I n}$. The bound (43) follows from these inequalities and (46).

Q. E. D.

Theorem 2. If the coefficients satisfy the conditions of Lemma 1, the difference system (4), (5), (18) has a unique solution.

Proof. Uniqueness is an immediate consequence of Lemma 1. Existence follows by the Fredholm alternative.

3. Generalizations. The restriction $c(x, y) \leqq 0$ can be removed as follows. Let $z_{\text {in }}$ satisfy (19). Then

$$
\zeta_{\text {in }}=e^{\lambda t_{n}} z_{\text {in }}
$$

satisfies (19) with $c_{\text {in }}$ replaced by

$$
c_{i n}^{*}=c_{i n}-a_{i n} \frac{e^{\lambda t_{n}}-e^{\lambda t_{n-1}}}{k},
$$

with $a_{i n}$ replaced by $e^{-\lambda h} a_{i n}$, and with each of $A_{i n}, B_{0 n}, B_{I n}$ multiplied by $e^{\lambda t_{n}}$.

If $c(x, y)$ is bounded, $\lambda$ can be chosen large enough so that

$$
\frac{e^{\lambda t_{n}}-e^{\lambda t_{n-1}}}{k}>\frac{1}{\alpha} \sup c(x, y)
$$

for all $k$ sufficiently small; in particular for $k<\frac{1}{4} \alpha \gamma^{-1}$. Thus $c_{i n}^{*}<0$. Therefore, Theorem 1 applies to $\zeta_{\text {in }}$, and, a fortiori, to $z_{\text {in }}$.

The arguments above can be extended to the problem, considered by Lotkin [5] and Isaacson [4], of the parabolic equation (1) in two regions $0<x<x_{0}$ and $x_{0}<x<1$, with conditions (2), (13) and

$$
\left\{\begin{array}{l}
u\left(x_{0}-, t\right)=u\left(x_{0}+, t\right) \\
\frac{\partial u}{\partial x}\left(x_{0}-, t\right)=\kappa(t) \frac{\partial u}{\partial x}\left(x_{0}+, t\right),
\end{array}\right.
$$

the derivatives in the second equation being replaced by either the centered differences (7) or the uncentered difference (16). An appropriate auxiliary function $\zeta$ 
can be constructed as in the proof of Lemma 2 if $\zeta^{*}$ in equation (33) is replaced by

$$
\zeta^{*}(x, t)= \begin{cases}x_{0}{ }^{2}(1-x)^{2}, & 0 \leqq x \leqq x_{0} \\ x^{2}\left(1-x_{0}\right)^{2}, & x_{0}<x \leqq 1\end{cases}
$$

4. The Non-Linear Problem. The results above can be extended to include the non-linear system

$$
\left\{\begin{array}{llr}
F\left(x, t, u, u_{x}, u_{x x}, u_{t}\right)=0, & 0<x<1,0<t \leqq T, \\
G\left(t, u, u_{x}\right) & =0, & x=0,0<t \leqq T, \\
H\left(t, u, u_{x}\right) & =0, & x=1,0<t \leqq T, \\
u(x, 0) & =f(x), & 0 \leqq x \leqq 1,
\end{array}\right.
$$

provided $F, G, H$, and $u$ satisfy certain continuity conditions. Indeed, if $u \varepsilon C^{4,2}([0,1] \times[0, T])$, then

$$
\left\{\begin{array}{lrr}
F\left(x, t, u, u_{x}, u_{x x}, u_{t}\right) & =F\left(x, t, u, \Delta_{x} u+\delta_{1}, \Delta_{x}^{2} u+\delta_{2}, \Delta_{t} u+\delta_{3}\right), \\
G\left(t, u, u_{x}\right) & =G\left(t, u, \Delta_{x}^{+} u+\delta_{4}\right), & 0<x<1,0<t \leqq T, \\
H\left(t, u, u_{x}\right) & =H\left(t, u, \Delta_{x}^{-} u+\delta_{5}\right), & x=0,0<t \leqq T,
\end{array}\right.
$$

where, for some constant $A$,

$$
\left\{\begin{aligned}
\left|\delta_{1}\right|,\left|\delta_{2}\right|,\left|\delta_{4}\right|,\left|\delta_{5}\right| & \leqq A h^{2} \\
\left|\delta_{3}\right| & \leqq A k
\end{aligned}\right.
$$

Let $w$, an approximation to $u$, satisfy

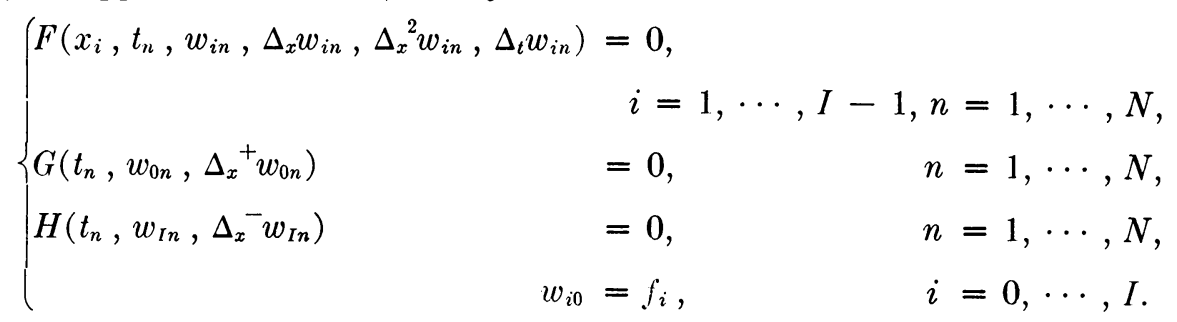

Suppose that $F, G$, and $H$ are continuous in $[0,1] \times[0, T]$, and that the derivatives $F_{3}, F_{4}, F_{5}, F_{6}, G_{2}, G_{3}, H_{2}$, and $H_{3}$ exist in $(0,1) \times(0, T)$. Then the mean value theorem applied to the difference of the respective equations in (53) and (55) yields

$$
\left\{\begin{array}{rr}
F_{3} \cdot\left(u_{i n}-w_{i n}\right)+F_{4} \cdot\left[\Delta_{x}\left(u_{i n}-w_{i n}\right)+\delta_{1}\right] & \\
+F_{5} \cdot\left[\Delta_{x}{ }^{2}\left(u_{i n}-w_{i n}\right)+\delta_{2}\right]+F_{6} \cdot\left[\Delta_{t}\left(u_{i n}-w_{i n}\right)+\delta_{3}\right]=0, & i=1, \cdots, I-1, \\
& n=1, \cdots, N, \\
G_{2} \cdot\left(u_{0 n}-w_{0 n}\right)+G_{3} \cdot\left[\Delta_{x}{ }^{+}\left(u_{0 n}-w_{0 n}\right)+\delta_{4}\right]=0, & n=1, \cdots, N, \\
H_{2} \cdot\left(u_{I n}-w_{I n}\right)+H_{3} \cdot\left[\Delta_{x}{ }^{-}\left(u_{I n}-w_{I n}\right)+\delta_{5}\right]=0, & n=1, \cdots, N, \\
\left(u_{i 0}-w_{i 0}\right) & =0,
\end{array}\right.
$$


where the values of the arguments of $F, G$, and $H$ lie between the values of the corresponding arguments in (53) and (55). Assume that all derivatives $F_{3}, F_{4}$, $F_{5}, F_{6}, G_{2}, G_{3}, H_{2}$, and $H_{3}$ are bounded, and that the relations

$$
\left\{\begin{array}{l}
F_{5}>0, \\
F_{6} \leqq-\alpha<0, \\
F_{5} \leqq \\
-G_{2}, G_{3},-H_{2},-H_{3} \geqq 0 \\
-G_{2}+G_{3} \geqq \delta>0 \\
-H_{2}-H_{3} \geqq \delta>0,
\end{array}\right.
$$

hold throughout $[0,1] \times[0, T]$. Then it is seen that equations (56) are identical with equations (19) (except that the coefficients now depend on $u$ and $w$ as well as $x$ and $t)$ and that Theorem 1 holds. Thus the error is $O\left(h^{2}+k\right)$.

5. Acknowledgment. The author wishes to thank Professor Jim Douglas for his many suggestions, particularly for his suggestion to use transformation (48).

Rice University

Houston, Texas

1. J. Douglas, JR., "On the numerical integration of quasi-linear parabolic equations," Pacific J. Math., v. 6, 1956, p. 35-42.

2. J. Douglas, JR., "Survey of numerical methods for parabolic differential equations," Advances in Computers, Vol. II, Academic Press, Inc., New York, 1961.

3. J. Douglas, JR., Unpublished Notes.

4. E. IsaAcson, "Error estimates for parabolic equations," Comm. Pure Appl. Math., v. 14,1961, p. $381-389$.

5. M. LoTKIN, "The numerical integration of heat conduction equations," J. Math. Phys., v. 37,1958, p. $178-187$.

6. M. E. Rose, "On the integration of non-linear parabolic equations by implicit methods," Quart. Appl. Math., v. 14, 1956, p. 237-248. 